\title{
Desvendando o Mistério do Duplo Cone
}

\author{
Uncovering the Mystery of the Double Cone \\ Alexandre Medeiros \\ Departamento de Física e Matemática, Universidade Federal Rural de Pernambuco, Recife, PE \\ Cleide Farias de Medeiros \\ Departamento de Educação, Universidade Federal Rural de Pernambuco, Recife, PE
}

Recebido em 25 de junho, 2003. Aceito em 29 de julho, 2003.

\begin{abstract}
Este artigo aborda a importância pedagógica de desvelar-se o mistério contido na aparente subida de um duplo cone. Apresentando, inicialmente, alguns detalhes históricos da criação deste instrumento, passamos à análise da própria origem física da referida ilusão de subida. Exibimos algumas figuras esclarecedoras da situação em foco e discutimos o relacionamento entre o deslocamento dos pontos de contato do instrumento com a rampa e o deslocamento do seu centro de massa. Em seguida procedemos a um equacionamento dos três ângulos principais envolvidos no problema. O texto conclui apontando para o fato de que mesmo um tal equacionamento não dá conta de toda a complexidade do mistério envolvido na subida do duplo cone. Para além das questões analisadas restam ainda outras ainda mais complexas a serem consideradas que apontam para problemas de estabilidade e de utilidade do instrumento.
\end{abstract}

This article approaches the pedagogical importance of uncovering the mystery involved in the apparent ascendant movement of a double cone. Initially presenting some historical details of the invention of such an instrument, we move onto the analysis of the very physical origin of that referred physical illusion. Some clarifying images are exhibited and the relationship between the motion of the points of contact between the cone and the ramp and the motion of its centre of mass is discussed. In the sequence, the mathematical relationship amongst the main angles involved in that situation is discussed. The text is concluded pointing out to the fact that even such a mathematization is not sufficient to account for all the complexity involved in that physical situation. Beyond the analysed questions rest some other issues even more complex than those to be considered pointing out to the stability problem and to the possible utility of the double cone.

\section{O Fascínio do Paradoxo do Du- plo Cone e a sua Importância Pe- dagógica}

Em artigo recente Greenslade Jr (1999) sugeriu a utilização de antigos artefatos experimentais como ferramentas propiciadoras de boas questões para o ensino da Física (Greenslade Jr, 1999). Se uma tal recomendação for levada a sério, poucos instrumentos prestar-se-iam tanto a uma tal finalidade quanto aqueles que encerram em seus funcionamentos uma certa dose de mistério. Dentre esses instrumentos misteriosos destaca-se o clássico experimento do avanço de um duplo cone em uma rampa inclinada. Extremamente popular, este experimento, ou simplesmente este brinquedo, é presença marcante em quase todos os laboratórios de Física escolares do mundo. O mistério que envolve o seu funcionamento consiste em um aparente desafio à lei da gravidade: colocado na parte mais baixa de uma rampa inclinada em formato de $\mathrm{V}$, o duplo cone parece subir a mesma
(Chagnon, 1993; Gardner, 1996; Malfi, 2003). Como surgiu esse interessante instrumento e qual a explicação para o inusitado fenômeno da sua aparente subida? Para responder a essas e a outras perguntas a respeito do estranho funcionamento do duplo cone vamos começar fazendo uma breve retrospectiva do seu surgimento histórico. Veremos que o fascínio que acompanha o funcionamento do duplo cone sempre esteve, desde o início, associado à motivação de platéias variadas com o intuito de despertar o seu interesse para as coisas da ciência. Hoje, como ontem, esse interesse despertado pelo comportamento inusitado do duplo cone pode servir de trampolim para que objetivos educacionais bem mais amplos possam vir a ser atingidos no ensino da Física, desde discussões filosóficas sobre a produção do conhecimento à veiculação de alguns conceitos físicos fundamentais na compreensão da natureza. É possível colocar em perspectiva a própria dependência do conhecimento produzido sobre este objeto em relação ao contexto de observação dos fenômenos em causa. Até mesmo a discussão de conceitos físicos como os de centro de massa, 
velocidade de rotação, estabilidade, momento angular, momento de inércia, oscilações forçadas, etc, pode vir a cena da discussão sobre o funcionamento deste fascinante instrumento. Vejamos, portanto, como toda essa misteriosa história começou.

As origens do duplo cone são incertas. Sua popularização certamente começa no século XVIII com George Adams, um fabricante inglês de instrumentos cient' $\cos$ e "los ócos". Embora esta denominação de "instrumentos los ó cos" possa parecer hoje um tanto anacrônica, os referidos instrumentos eram de fato artefatos que faziam pensar, como a bomba de vácuo, a máquina eletrostática de atrito, o mergulhador de Descartes e outros tantos que despertavam o fasc'nio das multidões que enchiam os salões para assistir as demonstrações dos seus efeitos misteriosos e espetaculares. A história desses artefatos, das suas origens e dos seus desenvolvimentos ocupa lugar de destaque nas próprias origens da história do ensino experimental da F'sica. O foco e o espaço deste artigo não permitem, entretanto, que nos alonguemos na discussão dos detalhes desse desenvolvimento mais amplo.

Importa-nos, entretanto, assinalar que mesmo antes da ampla divulgação do duplo cone feita por George Adams, que passou a fabricá-lo e comercializá-lo em larga escala a partir de 1759, um outro célebre demonstrador experimental, o francês Jean Theophile Desaguliers - já reivindicava para si a invenção do referido instrumento na primeira metade do século XVIII (1734).

Em suas próprias palavras, Desaguliers refere-se ao citado instrumento do seguinte modo: "Rombo cônico inventado, tal como o precedente, por mim, que tem, todavia, para cada vértice a maneira de dois cilindros juntos; $e$ duas réguas semelhantes as anteriores mas, contudo, mais compridas, por cima das quais o losango executa várias oscilações antes de descansar na posição mais baixa” (Desagulier, 1734, Tomo I, p. 55).

Alguns estudiosos têm levantado dúvidas sobre essa alegada autoria de Desaguliers, creditando a invenção desse instrumento, de modo impreciso aos membros da Accademia del Cimento. Isso remontaria a criação do duplo cone para os idos de 1657 a 1667 - per'odo durante o qual funcionou a referida Accademia. Entretanto, consultando-se os escritos da referida sociedade nada se encontra com referência ao duplo cone. Outros têm atribu'do a Galileu a criação do citado instrumento. Novamente, entretanto, não são encontrados registros entre os documentos de Galileu que creditem alguma veracidade a esta versão. Até prova em contrário, portanto, parece digna de crédito a alegação de autoria do instrumento reivindicada por Desaguliers. Esse artefato, de origem imprecisa, tem tido uma vida longa e ainda encanta, nos dias atuais, tantos quantos observam a sua intrigante "subida" pela rampa inclinada. Mas, como compreender tão inusitado comportamento?

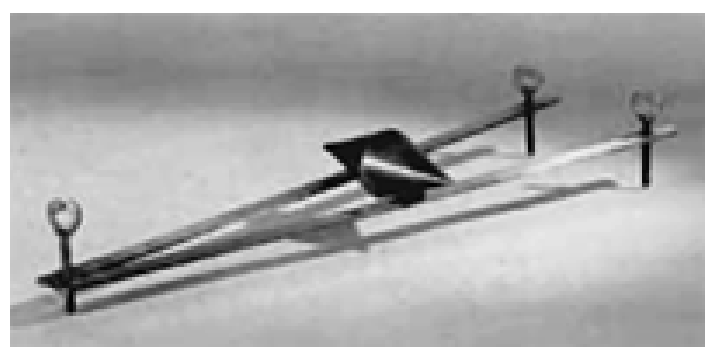

Figura 1. Duplo cone fabricado e comercializado por George Adams a partir de 1759 .

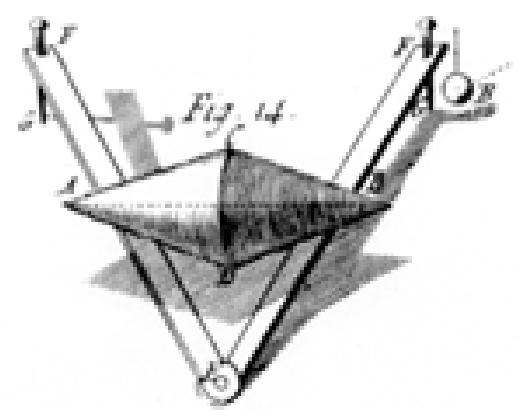

Figura 2. Duplo cone de Desaguliers

\section{A Complexidade do Fenômeno e a Origem da Ilusão da Subida do Du- plo Cone}

A maior parte dos textos escritos sobre o comportamento misterioso do duplo cone parece contentar-se simplesmente em assinalar que a tão decantada "subida" do artefato tratarse-ia apenas de uma mera ilusão de ótica e que seria, em verdade, o centro de massa do duplo cone que estaria necessariamente descendo em um tal movimento. Tomemos alguns exemplos de tais explicações:

"Você car á surpreso ao ver o duplo cone rolar ladeira acima ao longo da rampa. Mas, realmente, sobe? Olhe para as extremidades do cone. Elas descem e assim também o próprio duplo cone realmente desce. Mas ele 'parece' estar desa ando a lei da gravidade. Que alguma coisa poderia rolar ladeira acima é algo verdadeiramente altamente inesperado e realmente imposs'vel, a menos que esta coisa tenha a velocidade necessária. O que nós vamos fazer agora não é tornar o imposs'vel realmente poss'vel, mas fazer o imposs vel parecer poss'vel" (De Vries, 19 72, p. 55).

"Os cones não estão desa ando a lei da gravidade. $\mathrm{Na}$ verdade, quando os cones se movem o seu 'centro de gravidade' (o ponto em relação ao qual o peso está igualmente distribu'do) move-se para baixo. Note que o centro de massa dos dois cones aproxima-se do chão quando ele se move ao longo das rampas" (VanCleave, 1991, pp. 68-69). 
Ainda que possamos admitir que tal fato realmente ocorre - a descida efetiva do centro de massa do objeto esta afirmativa, entretanto, está longe de ser trivial.

Em primeiro lugar, a sua constatação encerra um olhar intencionalmente direcionado para determinados aspectos do movimento do objeto em causa. Precisamos, portanto, deixar claro que olhar é esse que nos permite observar a descida do centro de massa do duplo cone. Em segundo lugar, ainda que o observador possa perceber que de fato o centro de massa do duplo cone desce, isso não tira, de imediato, a aura de mistério que envolve o instrumento. Afinal, mesmo aceitando-se que o centro de massa do instrumento desce a rampa inclinada em $\mathrm{V}$, uma questão ainda permanece: por que temos a clara impressão de que o duplo cone sobe? Em outras palavras: de onde nasce a ilusão da subida que parece resistir tão tenazmente diante dos nossos olhos atentos? E em terceiro lugar, qual a condição para que uma tal "ilusão" se processe?

Tentemos responder, conjuntamente, a essas três questões levantadas.

Algo que precisa ser analisado com cuidado é que, de fato, alguns pontos, que atraem a nossa atenção no fenômeno observado, realmente se elevam com o avanço do cone. Que pontos são esses e como esse paradoxo pode ser contornado?

Os pontos que vemos subir são os pontos de contato entre o duplo cone e a rampa. Eles, de fato, vão se tornando mais altos à proporção que o cone avança pela rampa. É fundamental, porém, que se percebam duas coisas muito importantes para a elucidação do aparente paradoxo:

1. Os pontos de contato não são sempre os mesmos, pois o cone está girando e não deslizando sobre a rampa. Eles são pontos que estão continuamente variando; não são pontos fixos do duplo cone;

2. Para desvendar o aparente paradoxo do duplo cone é preciso compreender, também, qual a condição física que precisa ser satisfeita para que tal ilusão de subida ocorra.

Como notam, apropriadamente, Carpenter Jr \& Minnix (1993): "Quando o duplo cone é colocado sobre a rampa ele rola em direção oposta àquela que rolaria um cilindro porque ao rolar o seu centro de massa, na verdade, desce enquanto os seus pontos de contato com os lados da rampa sobem" (p. M-482; grifo nosso). Isso pode ser percebido na figura abaixo, onde representamos lateralmente o avanço de um duplo cone.

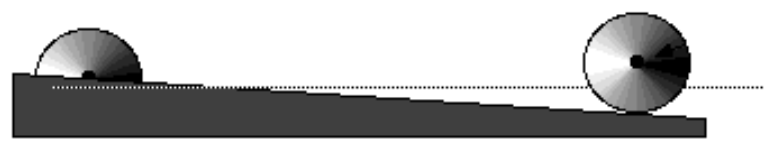

Figura 3. Atentando-se para o eixo do duplo cone pode-se perceber a sua descida quando o mesmo avança da direita para a esquerda em uma aparente "subida".

Mesmo com o auxílio da figura anterior, entretanto, somos forçados a admitir que a ilusão de subida ainda parece um tanto persistente. Tentemos, portanto, observar o fenômeno do avanço do duplo cone sob outros ângulos na tentativa de clarificar o paradoxo. Os diagramas seguintes podem nos dar uma visão de que, mesmo sob uma observação lateral, é possível compreender melhor a natureza do problema a ser percebido. A figura seguinte da esquerda é um esquema no qual estão ressaltados dois pontos principais na compreensão do fenômeno em causa: o centro de massa e os pontos de contato do cone com a rampa. Nela pode-se observar, talvez com maior facilidade, que os pontos de contato de fato sobem - apesar de não serem sempre os mesmos - enquanto o centro de massa do objeto desce. A ilusão da subida provém, justamente, de fixarmos nossa atenção nos referidos pontos de contato. A figura abaixo à direita é um esquema representando o mesmo duplo cone agora com um disco circular - de mesmo raio do cone - pregado em cada um dos vértices. Deste modo, tudo que pode ser observado é o avanço do disco e o que acontece com o prego colocado no centro do mesmo. Embora se trate da mesma situação anterior, o fato de camuflarmos a superfície cônica elimina a ilusão da subida. O que vemos, realmente, é simplesmente o avanço do disco - que esconde o cone - da direita para a esquerda e a descida do seu centro geométrico.

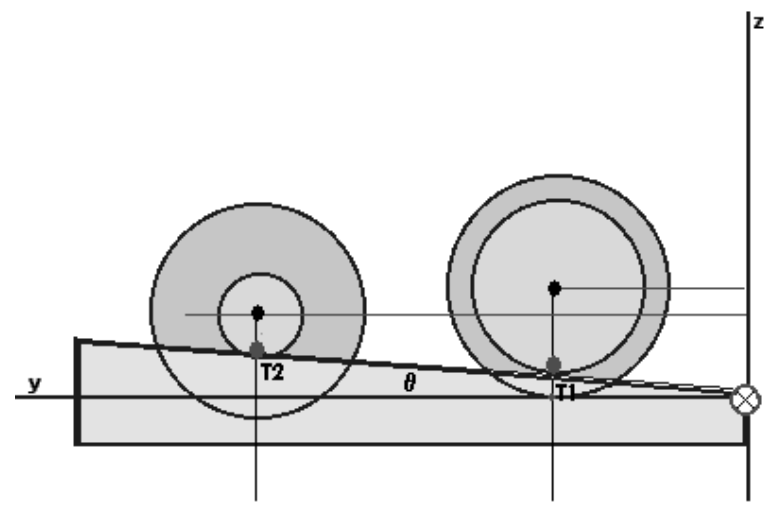

Figura 4. Os pontos de contato de fato sobem - apesar de não serem sempre os mesmos.

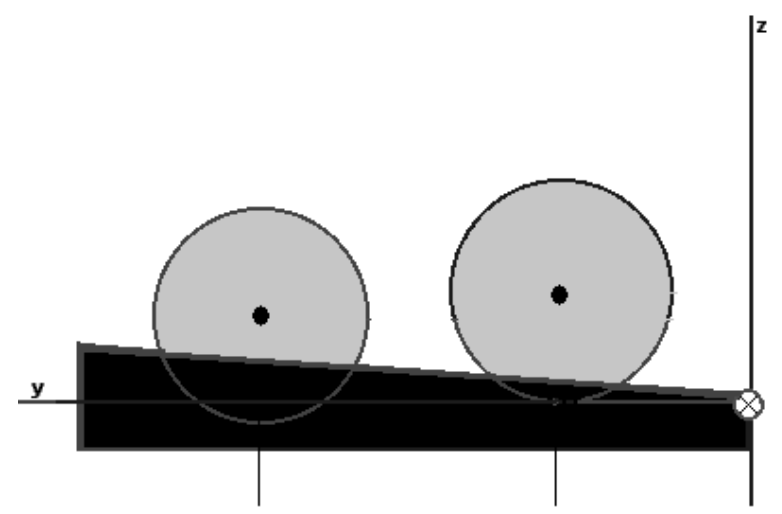

Figura 5. O fato de camuflarmos a superfície cônica elimina a ilusão da subida.

Um outro olhar, em perspectiva, pode contribuir ainda mais para elucidar a questão. A figura seguinte pode revelar o quanto é enganoso para o nosso olhar fixarmos a atenção 
exageradamente no que ocorre com os pontos de contato entre o cone e as rampas. A figura tenta revelar, de outro ângulo, a essência do problema: enquanto os pontos negros centrais - o centro de massa do duplo cone - descem, os pontos laterais, que representam os pontos de contato entre o cone e as rampas, efetivamente sobem quando o objeto avança da esquerda para a direita.

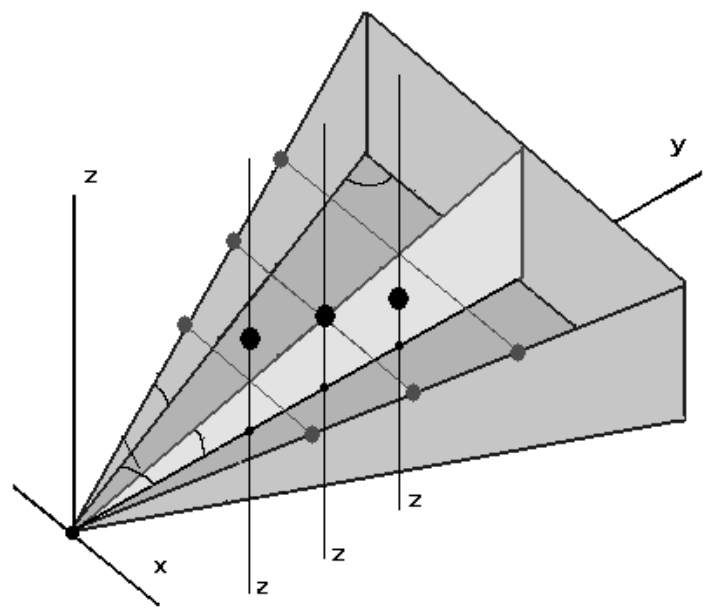

Figura 6. Vista superior em perspectiva dos deslocamentos do centro de massa e dos pontos de contato.

Entretanto, mesmo admitindo-se que as figuras anteriores já sejam suficientes para elucidar o desencontro entre a descida do centro de massa do duplo cone e a subida dos pontos de contato deste com as rampas, resta ainda, talvez, uma outra inquietação. A questão a ser devidamente considerada é a seguinte: como pode haver tal desencontro? Não é psicologicamente confortável simplesmente saber da existência de um tal desencontro. É lícito afirmar que muitos daqueles indivíduos que olham atentamente o comportamento misterioso do duplo cone ainda ficam em dúvida após uma explicação do tipo daquela montada nas figuras anteriores. E, infelizmente, isso, quase sempre, é tudo que é fornecido para elucidar a questão. O que é de suma importância e, portanto, precisa ficar muito claro é que os pontos de contato não são sempre os mesmos. Isso elimina o aparente paradoxo de um certo ponto de um corpo sólido estar constantemente subindo - o ponto de contato - enquanto um outro - o centro de massa - está constantemente descendo. Mais uma vez recorremos ao inestimável auxílio cognitivo das imagens para a compreensão da situação em causa. Observe-se, desta vez, o avanço do cone visto de cima bi-dimensionalmente. A figura da esquerda põe em destaque o que ocorre com os pontos de contato entre o cone e a rampa quando este avança da esquerda para a direita. Importa-nos, agora, perceber que não sendo esses pontos sempre os mesmos o seu lugar geométrico é uma espiral traçada sobre o cone a partir do vértice. Na foto à direita colocamos uma seqüência de pontos pondo este fato em evidência. Fica, portanto, esclarecida, a primeira questão geralmente colocada nas explicações com uma aparência ilusória de trivialidade. Resta, entretanto, a outra questão a respeito da condição física a ser respeitada para que tal ilusão de subida do cone ocorra. Sem responder a essa questão estaremos muito longe ainda, de compreendermos o problema.

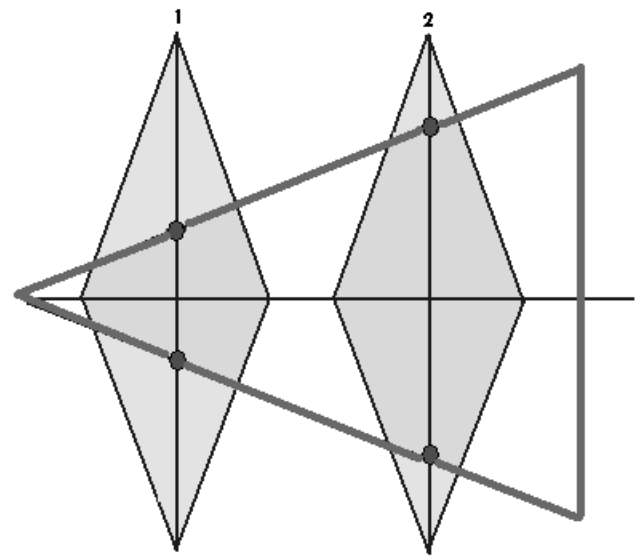

Figura 7. O que ocorre com os pontos de contato entre o cone e a rampa quando este avança da esquerda para a direita.

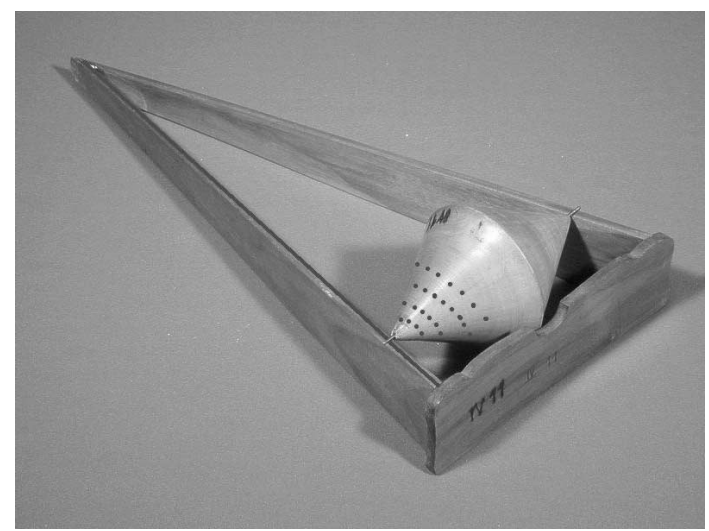

Figura 8. O lugar geométrico dos pontos de contato entre o duplo cone e a rampa é a espiral formada com o avanço do aparato.

\section{Algumas Explicações Parciais do Fenômeno}

As tentativas de geometrização da situação física do duplo cone podem assumir várias versões. Algo fundamental, em todas elas, entretanto, é a compreensão de que os ângulos de subida das rampas e de abertura das mesmas jogam um papel primordial na matematização da situação física em causa. Observando-se as figuras representativas dos duplo cones de Desaguliers e de Adams - contidas no início deste artigo pode-se perceber que a presença de parafusos reguladores da abertura e da inclinação das rampas já denotava um estado de alerta sobre a importância daqueles dois ângulos na compreensão do fenômeno. Levando em conta estes mesmos ângulos de abertura e de inclinação das rampas, Canalle \& Moura (1998) apresentam uma explicação matemática para o paradoxo do duplo cone que consiste, basicamente, 
em relacionar tais ângulos com as distâncias relevantes na situação em causa. Estes autores desenham o duplo cone em corte lateral sobre uma rampa e colocam, também, em destaque os dois ângulos em causa (inclinação das rampas e abertura das mesmas), segundo as figuras abaixo.

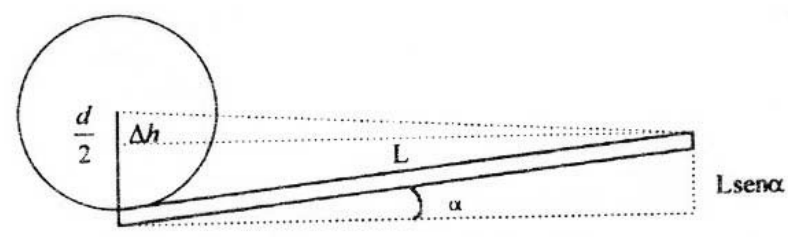

(a)

Figura 9. Vista lateral do duplo cone.

Canalle \& Moura (1998, p 327) argumentam que o centro de massa do duplo cone precisa cair de uma altura:

$\Delta h=\frac{d}{2}-L \operatorname{sen} \alpha>0 \Rightarrow \frac{d}{2}>L \operatorname{sen} \alpha$

"Mas o comprimento L do suporte e o ângulo $\beta$ da abertura do suporte (figura $b$ ) estão relacionados por":

$\operatorname{sen} \beta=\frac{H}{2 L} \Rightarrow L=\frac{H}{2 \operatorname{sen} \beta}$

Substituindo a segunda expressão na primeira, os autores encontram a relação entre o diâmetro e o comprimento do duplo cone e os ângulos de abertura e de inclinação das rampas:

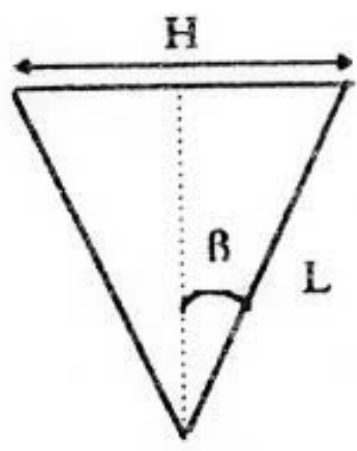

(b)

Figura 10. Vista superior da rampa em V.

$$
\frac{\operatorname{sen} \alpha}{\operatorname{sen} \beta}<\frac{d}{H}
$$

A questão central que permanece, entretanto, sem discussão é que esses dois ângulos apesar de serem necessários, não são suficientes para equacionar o problema. De fato, sem considerar, também, o próprio ângulo de geração do duplo cone o problema fica inconcluso. Um tratamento mais geral do problema, incluindo os três ângulos mencionados é feito por Ferraz Neto (2002). Infelizmente, a abordagem por ele adotada não toma como início da solução a condição física a ser satisfeita, qual seja a de que a ilusão da subida do instrumento será produzida sempre que a distância vertical descida pelo seu centro de massa for maior que aquela que sobem os pontos de contato entre o cone e as rampas. Procedendo por uma geometrização inicial ele impõe a condição física apenas em meio à referida geometrização do problema. Isso dificulta sobremaneira a visualização, pelo iniciante, da solução encaminhada para a questão. Por isso, seguimos neste artigo o rumo traçado na abordagem clássica do problema apresentada por Mitrofanov (1980) na revista russa "Kvant" e traduzida para o inglês na revista americana "Quantum" (1996). Esta abordagem foi igualmente comentada de modo muito pertinente por Rizzi (1981) na revista italiana "Física nella Scuola". Apresentamos, em seguida, de forma mais comentada, essa referida linha de abordagem do problema.

\section{Geometrizando a Condição Física da Produção da Ilusão da Subida}

A condição para a produção da ilusão de subida do duplo cone, como vimos, é que a distância que o centro de massa do duplo cone desce ao avançar pela rampa $(H)$ seja maior que a distância subida pelo ponto de contato entre o cone e rampa $(h)$. Em termos simbólicos, temos que:

$$
H>h
$$

Expressemos essa desigualdade em função dos três ângulos notáveis na situação estudada, a saber:

$\alpha$ - o ângulo entre o plano das rampas do duplo cone e o plano horizontal; $2 \beta$ - o ângulo formado entre as rampas $2 \gamma$ - o ângulo de geração do duplo cone; formado no vértice do mesmo.

A Fig. 11, representa um duplo cone. Colocando-o sobre a rampa na posição 1 ele se desloca em direção à posição 2.

Pode-se notar neste avanço que o seu centro de massa desce de uma distância $H$ dada por:

$$
H=\frac{l_{2}}{2} \operatorname{tg} \gamma-\frac{l_{1}}{2} \operatorname{tg} \gamma
$$

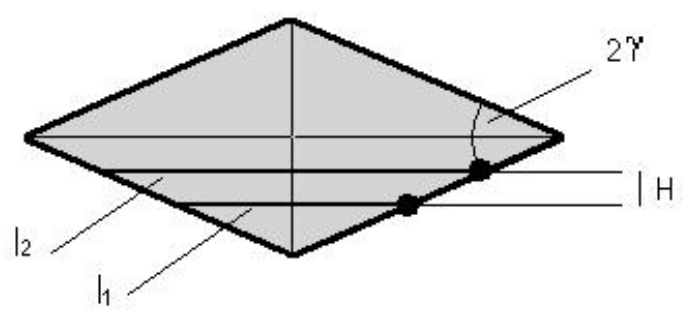

Figura 11. Duplo cone com os pontos de contato em destaque.

Ou seja:

$$
H=\frac{l_{2}-l_{1}}{2} \operatorname{tg} \gamma
$$

A Fig. 12 representa uma rampa inclinada sobre a qual o duplo cone é colocado. 
Podemos perceber que a distância $h$ subida pelo ponto de contato pode ser escrita como:

\section{$h=M N \operatorname{sen} \alpha$}

Mas podemos observar que $M N$ é dado por: $M N=\frac{l_{2}}{2} \cot g \beta-\frac{l_{1}}{2} \cot g \beta$

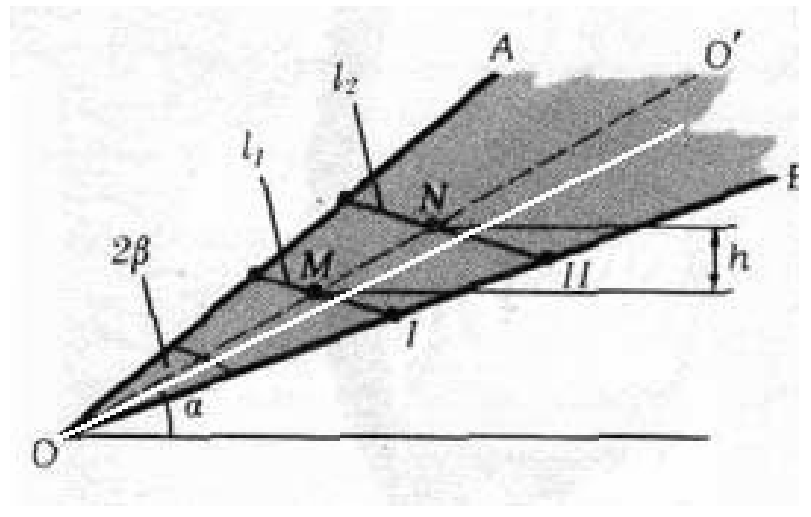

Figura 12. esquema da rampa com os ângulos principais.

Ou seja:

$$
M N=\frac{l_{2}-l_{1}}{2} \cot g \beta
$$

Assim, teremos para $h$ o valor:

$$
h=\frac{l_{2}-l_{1}}{2} \cot g \beta \operatorname{sen} \alpha
$$

Deste modo, a condição física para que a ilusão de subida do duplo cone seja produzida, dada pela desigualdade $H>h$, fica sendo:

$$
\frac{l_{2}-l_{1}}{2} \operatorname{tg} \gamma>\frac{l_{2}-l_{1}}{2} \cot g \beta \operatorname{sen} \alpha
$$

Ou seja:

$$
\operatorname{tg} \gamma>\cot g \beta \operatorname{sen} \alpha
$$

donde:

$$
\frac{\operatorname{tg} \gamma}{\cot g \beta}>\operatorname{sen} \alpha \Leftrightarrow \operatorname{tg} \gamma \operatorname{tg} \beta>\operatorname{sen} \alpha
$$

Logo:

$$
\operatorname{sen} \alpha<\operatorname{tg} \beta \operatorname{tg} \gamma
$$

que é, portanto, a condição a ser satisfeita pelos três ângulos para que a ilusão de subida do duplo cone pela rampa inclinada venha a ser produzida.

É fácil mostrar-se que se

$$
\operatorname{sen} \alpha .>\operatorname{tg} \beta \operatorname{tg} \gamma
$$

o duplo cone descerá a rampa, e se:

$$
\operatorname{sen} \alpha .=\operatorname{tg} \beta \operatorname{tg} \gamma
$$

o duplo cone estará em equilíbrio indiferente.

\section{Conclusões}

A primeira conclusão a ser tirada dos argumentos expostos neste artigo é a de que a tentativa de desvendar o mistério da aparente subida do duplo cone não se esgota simplesmente em afirmarmos que é o centro de massa do instrumento que está descendo. Mesmo a simples percepção deste fato parece necessitar da mediação de determinadas imagens nem sempre convenientemente relevadas pelos educadores. Por outro lado, a explicitação da condição física para que a subida seja produzida parece algo também não trivial que necessita, igualmente, ser mediada por alguém mais experiente para que o iniciante compreenda o seu sentido físico. De outra parte, o equacionamento de tal condição, explicitando-a em função dos três ângulos notáveis do problema, parece ser uma condição necessária, mas não suficiente para desvendar todo o mistério do duplo cone. Mas, se o equacionamento referido não é suficiente para revelar toda a riqueza e o potencial pedagógico do duplo cone, o que mais poderia ser investigado no mesmo?

Há muito mais coisas a serem exploradas no duplo cone do que aquilo que o espaço reduzido deste artigo permitiria analisar. Para começar, poder-se-ia perguntar, por exemplo: o que ocorre ao invertermos a inclinação das rampas? Poderíamos questionar também, o que aconteceria se o duplo cone fosse formado pela junção dos vértices e não das suas bases. E mais ainda: o que dizer da estabilidade do sistema relacionada à velocidade dos vários pontos do instrumento, ao seu momento de inércia e ao seu momento angular? Isso e muito mais poderia conduzir a uma outra questão básica: seria o duplo cone um mero brinquedo ou a sua Física encerraria também alguma aplicação útil?

A resposta a todas essas questões, entretanto, ensejaria a elaboração de um outro artigo, pois pela sua complexidade inerente o mistério do duplo cone continua vivo.

De todo modo, pelo fascínio do seu aspecto misterioso e pela riqueza da Física nele envolvida, o duplo cone constitui-se realmente em um "instrumento filosófico" nem sempre devidamente valorizado. A sua utilização pedagógica poderia ser feita em diversos níveis de aproximação sintonizados com os níveis dos estudantes. Assim, a riqueza de situações nele envolvidas forma um arco bastante amplo que poderia, portanto, ser explorado desde o ensino fundamental até a universidade.

Créditos sobre as figuras: fig 1: Science Museum (London); fig 2: Desaguliers, 1734, Tomo I, p. 55; fig 3: University of Maryland; fig 4, fig 5, fig 6 e fig 7: baseadas em outras semelhantes encontradas em Ferraz Netto (2002); fig 8: fotografia de pontos desenhados sobre duplo cone Scienco; fig 9 e fig 10: Canalle \& Moura, 1998, p.327; fig 11: baseada em outra semelhante presente em Mitrofanov, 1980, p.24; fig 12: originalmente presente em Mitrofanov, 1980, p.25. 


\section{Referências}

CANALlE, J. \& MOURA, R. Duplo Cone, Quádrupla Finalidade. Caderno Catarinense de Ensino de Física; vol. 15, n.3, pp 323-327, Dezembro de 1998.

CARPENTER JR, D. \& MINNIX, R. 'Double Cone - High Wire Toy' In Dick and Rae Physics Demo Notebook. P. M482, Dick and Rae Inc, Lexington, Virginia, 1993.

CHAGNON, P. Animated Displays III: Mechanical Puzzles, The Physics Teacher; vol. 31, pp. 32-37, Jan 1993.

DESAGULIERS, J. A Course of Experimental Philosophy. Londres, Tomo I, p. 55, Pl. IV, Fig. 14, 1734.

DE VRIES, L. The Book of Experiments. Carousel Books, London, 1972.

FERRAZ NETO, L. Paradoxo Mecânico: Duplo Cone. Publicação on line no site Feira de Ciência. http://www.feiradeciencias.com.br/sala06/06_08.asp.
Acessado em 10 de novembro de 2002.

GARDNER, M. The Ball that Rolls Uphill. The Physics Teacher, vol. 34, n.7, p. 461, October 1996.

GREENSLADE JR, T. Examination Questions Based on Historical Apparatus. The Physics Teacher; vol. n.3, March 1999, pp. 172-173.

MALFI, P. Apparato per il Cosiddetto Paradosso Meccanico. Liceo Foscarini di Venezia: Museo di Física a Venezia, Veneza, 2003.

MITROFANOV, A. Up the Down Incline: Gravity Defied? or Obeyed Unusually? Quantum; vol 2, n2, March-April 1996, pp. 44-45. Tradução do artigo originalmente publicado em russo na revista Kvant; vol 11, n2, 1980, pp. 24-25. RIZZI, J. In Salita Lungo un Piano Inclinato. La Fisica nella Scuola; vol. 14, n.1, pp.50-51, 1981.

VANCLEAVE, J. Up Hill In Physics for Every Kid. John Wiley \& Sons Inc, New York, 1991, pp. 68-69. 